\title{
A PESQUISA NA ÁREA DA ENFERMAGEM ONCOLÓGICA: UM ESTUDO DAS PUBLICAÇÕES EM PERIÓDICOS NACIONAIS ${ }^{1}$ RESEARCH IN ONCOLOGICAL NURSING: A STUDY OF THE PUBLICATIONS IN NATIONAL PERIODICALS \\ LA INVESTIGACIÓN EN EL ÁREA DE LA ENFERMERÍA ONCOLÓGICA: UN ESTUDIO DE LAS PUBLICACIONES EN PERIÓDICOS NACIONALES
}

\author{
Marléa Chagas Moreira², Teresa Caldas Camargon, Vilma de Carvalho ${ }^{4}$, Carolina Farias de Figueirêdo, \\ Luciana Dias da Rosa', Mônica de Fátima Bolzan
}

${ }^{1}$ Trabalho apresentado no 13th European Câncer Conference. Paris, outubro 2005.

${ }^{2}$ Doutora em Enfermagem. Professora Adjunto na Escola de Enfermagem Anna Nery da Universidade Federal do Rio de Janeiro (EEAN/UFRJ). Líder do Grupo de Pesquisa em Gerência e Processo de Cuidar na Enfermagem em Oncologia.

${ }^{3}$ Doutora em Enfermagem. Enfermeira de Educação Continuada no Instituto Nacional do Câncer - HCIII. Líder Grupo de Pesquisa em Gerência e Processo de Cuidar na Enfermagem em Oncologia.

${ }^{4}$ Docente Livre. Professora Emérito da UFRJ. Líder do Grupo Linha de Pesquisa e Estudos Epistemológicos para a Enfermagem. Pesquisadora do Conselho Nacional de Desenvolvimento Científico e Tecnológico (CNPq).

${ }^{5}$ Residente em Enfermagem. Membro do Grupo de Pesquisa em Gerência e Processo de Cuidar na Enfermagem em Oncologia.

PALAVRAS-CHAVE: Enfermagem oncológica. Pesquisa em enfermagem. Conhecimento.
RESUMO: Trata-se de um estudo de classificação da produção do conhecimento na enfermagem em oncologia cujos objetivos foram: identificar as publicações na enfermagem em oncologia no período de 1994 a 2004 e analisar nas publicações, os aspectos epistemológicos destacados nas temáticas focalizadas e no enquadramento teórico-metodológico adotado nos estudos. O referencial teórico-metodológico está aliado a uma metodologia de categorização epistemológica para a pesquisa na enfermagem. Utilizou-se como fontes de dados três periódicos: a Revista Brasileira de Enfermagem, a Revista Latino-Americana de Enfermagem e Revista Brasileira de Cancerologia. Com base nos resultados, as autoras consideram que o conhecimento produzido, na área de enfermagem oncológica, é consistente com a complexidade e tentativas de explicação sobre a arte de cuidar dos clientes e com esforços de ampliar critérios e padrões assistenciais.
KEYWORDS: Oncologic nursing. Nursing research. Knowledge.

\begin{abstract}
This is a classification study of knowledge production in Oncological Nursing. The objectives are: to identify the publications in Oncological Nursing in the period between 1994 and 2004, and to analyze in these publications the epistemological aspects highlighted in the focused themes as well as the theoretical-methodological inclusion adopted in the studies. The theoretical-methodological reference is allied to an epistemological categorization methodology for nursing research. Three periodicals were used as data sources: the Brazilian Journal of Nursing, the Latin American Journal of Nursing and Brazilian Journal of Cancerology. Based on the results, the authors consider that the knowledge produced in oncological nursing, is consistent with the complexity and attempts to explain the art of taking care of the customers, and with efforts to extend assistance criteria and standards.
\end{abstract}

PALABRAS CLAVE: Enfermería oncológica. Investigación en Enfermería. Conocimiento.
RESUMEN: Estudio de clasificación sobre la producción del conocimiento en la enfermería en oncología. Los objetivos son: identificar las publicaciones en la enfermería en oncología en el período entre 1994 y 2004 y, analizar en las publicaciones, los aspectos epistemológicos señalados en las tematicas focalizadas y en el encuadramiento teorico-metodológico adoptado en los estudios. El referencial teorico-metodológico conjuntamente a una metodología epistemológica para la investigación en la enfermería. Fueram utilizados como fuentes de datos tres periódicos: la Revista Brasileña de Enfermería, la Revista Latinoamericana de Enfermería y la Revista Brasileña de Cancerología . De acuerdo con los resultados, las autoras consideran que el conocimiento producido en el área de la enfermería oncológica éstos son consistentes con la complejidad y las tentativas de explicación sobre el arte de cuidar de los clientes y con los esfuerzos de ampliar los criterios y los patrones asistenciales.
Endereço: Marléa Chagas Moreira

R. Abelardo Barbosa, 14

28.990-000 - Itaúna, Saquarema, RJ.

E-mail: marleachagas@gmail.com
Artigo original: Pesquisa Recebido em: 22 de maio de 2006. Aprovação final: 30 de outubro de 2006. 


\section{INTRODUÇÃO}

Este estudo está inserido no projeto integrado de pesquisa "A produção de conhecimento na enfermagem em oncologia: bases para modelos gerenciais e assistenciais", em desenvolvimento no contexto do Grupo de Pesquisa Gerência do Processo de Cuidar na Enfermagem em Oncologia e Grupo Linha de Pesquisa e Estudos Epistemológicos para a Enfermagem, registrados na Universidade Federal do Rio de Janeiro (UFRJ) e cadastrados no Diretório dos Grupos de Pesquisa no Brasil, Conselho Nacional de Desenvolvimento Científico e Tecnológico (CNPq).

A idéia principal, a nortear o estudo, visa principalmente o interesse da enfermagem no que concerne à especificidade do conhecimento produzido quanto às dimensões da arte de cuidar e formalidades da atuação, isto é, modus operandi, de enfermeiras(os). Especificamente, procurou-se buscar respostas consistentes com o saber da enfermagem na área de oncologia, um saber mais voltado para a internalidade epistemológica da profissão e dirigido à prática pedagógica e assistencial.

A produção de conhecimento tem sido uma preocupação constante na trajetória evolutiva $\mathrm{da}$ história da enfermagem mundial, evidenciada mais enfaticamente no Brasil, nos últimos trinta anos, com a implementação dos cursos de pós-graduação "stricto sensu" (mestrado e doutorado). Essa preocupação reflete, ao nosso ver, o empenho das enfermeiras em inserir no âmbito de suas ações a arte e a ciência, para enfrentar os desafios impostos pelas transformações científicas, tecnológicas e políticas contemporâneas, bem como saber ou conhecer mais sobre a enfermagem e seus termos teóricos e práticos, valorizar-se nas relações com os assistidos e seus pares, e identificar-se como profissão no mundo. ${ }^{1}$

Pode-se dizer que tal preocupação leva-nos a pensar o conhecimento produzido pela enfermagem a partir de dois aspectos. De um lado, a importância de uma atitude crítica que releve o papel social do pesquisador, o que implica integrar a ciência aos processos políticos e sociais de cada sociedade, a partir de reflexões acerca dos pressupostos, resultados, alcance, limites e significações sócio-culturais da atividade científica desenvolvida. ${ }^{2-3} \mathrm{E}$, de outro, a noção bachelariana de que o pensamento científico não é um sistema acabado, de dogmas evidentes, mas uma incerteza motivadora para o cientista. Tal noção dá ao conhecimento produzido um caráter operativo, de transformação e movimento, em consonância com a evolução do planeta. ${ }^{2}$
Ao longo de nossa experiência como enfermeiras docentes e assistenciais, a preocupação com o conhecimento aliada às atividades desenvolvidas no cotidiano da prática de cuidar, administrar, ensinar, e pesquisar na área de oncologia, nos dão apoio à idéia da amplitude e complexidade que envolve a temática do conhecimento pertinente à enfermagem oncológica e à tendência da produção científica na área.

Para delinear o estudo elaborou-se como objetivos: identificar as publicações em enfermagem oncológica no período de 1994 a 2004 e analisar nas publicações, os aspectos epistemológicos destacados nas temáticas focalizadas e no enquadramento teórico-metodológico adotado.

A opção por estudar as publicações dessa década decorre dos resultados de estudo realizado acerca das tendências da produção de conhecimento na enfermagem oncológica no Brasil, que revelou o impulso da produção acadêmica nacional, na área, a partir da década de noventa do século passado. ${ }^{4}$

\section{BASES TEÓRICO-METODOLÓGICAS}

O referencial teórico-metodológico deste estudo está aliado a uma metodologia de categorização epistemológica para a pesquisa na enfermagem. ${ }^{5}$

Tal metodologia estruturada a partir de elementos de enfoque epistemológico, configura-se como um esquema arquetípico, ou seja, um instrumento de análise estruturado a partir dos seguintes aspectos: 1) constituição do tema ou problema da pesquisa, que possibilita identificar o tipo de contribuição para a enfermagem no que se refere à construção do conhecimento; 2) enquadramento teórico-metodológico, apresentando os quadros referenciais que orientam a produção do conhecimento; 3) dados e/ou resultados, que permitem apontar as estratégias adotadas para descrição da realidade e análise/discussão da situação problema; e 4) aspectos epistemológicos destacados, relativos à situação do conhecimento produzido, destacando a intencionalidade objetiva do pesquisador. ${ }^{5}$

Para o estudo optou-se por realizar uma investigação de natureza quantitativa, com indicativos qualitativos, a partir de análise documental. Foram utilizadas como fontes de dados artigos publicados no período de 1994 a 2004 em três periódicos: a Revista Brasileira de Enfermagem (REBEn), a Revista Latino-Americana de Enfermagem (Rev Latino-Am. Enferm.) e Revista Brasileira de Cancerologia (RBC). A opção pelos periódicos de enfermagem justifica-se 
pela representatividade, regularidade, abrangência das opções temáticas para publicação, além da classificação na indexação CAPES; a RBC foi selecionada por ser órgão oficial de divulgação da produção científica nacional de oncologia, inclusive da Sociedade Brasileira de Enfermagem Oncológica.

Para seleção dos artigos, localizados a partir de busca manual nos periódicos, foi utilizado como critério de inclusão aqueles configurados nas categorias pesquisa, revisão, relato experiências/caso e reflexão.

Dos 92 artigos identificados, 04 foram excluídos por não apresentar os requisitos necessários à classificação. A amostra constituiu-se de 88 artigos dos quais 16 da REBEn, 25 da Rev Latino-Am. Enferm. e 47 da RBC.

Para a classificação do material foi realizada leitura analítica dos artigos e organização dos dados a partir de roteiro estruturado com base nos aspectos que compõem o esquema arquetípico proposto na metodologia de categorização epistemológica para a pesquisa na enfermagem. ${ }^{5}$ Os dados foram quantificados e analisados de acordo com literatura concernente às tendências e prioridades de pesquisa na enfermagem e na especificidade da oncologia, bem como de reflexões e críticas das autoras.

\section{APRESENTAÇÃO DOS RESULTADOS}

Os resultados serão apresentados em duas categorias: 1) questões epistemológicas relacionadas às temáticas estudadas; e 2) questões epistemológicas relacionadas ao enquadramento teórico-metodológico.

\section{Questões epistemológicas relacionadas às temáticas estudadas}

Neste item são apresentadas as questões epistemológicas destacadas a partir da constituição do tema ou problema de pesquisa. Foram considerados, além da configuração dos estudos, os temas focalizados e a área que delimita o ato do conhecer, ou seja, a setorização para contribuição do conhecimento produzido. Possibilitou a apreensão dos fenômenos prioritários para investigação na enfermagem oncológica, sinalizando a intencionalidade objetiva dos pesquisadores.

Os resultados evidenciaram que houve regularidade de publicações no período estudado, com aumento da produção a partir de 2002, conforme pode ser observado na Figura 1.
Entendemos que esses resultados refletem os desafios e empreendimento das enfermeiras brasileiras para o fortalecimento da pós-graduação em enfermagem, na busca pela cientificidade. Nesse sentido vale ressaltar três aspectos que influenciaram esse panorama: 1) após a década de noventa houve expressivo crescimento dos programas de pós-graduação em enfermagem no país, apesar das disparidades regionais; 2) no decorrer desse período as escolas intensificaram políticas para qualificação do corpo docente; e, 3) como elemento determinante desse processo, as mudanças operadas no sistema de avaliação da Coordenação de Aperfeiçoamento de Pessoal de Nivel Superior (CAPES), principalmente a partir do biênio 96/97, quando padrões internacionais de nível de excelência foram tomados como referência. ${ }^{6}$ Assim as avaliações dos programas tornaram-se essencialmente acadêmicas, valorizando, sobretudo, a pesquisa e as publicações científicas.?

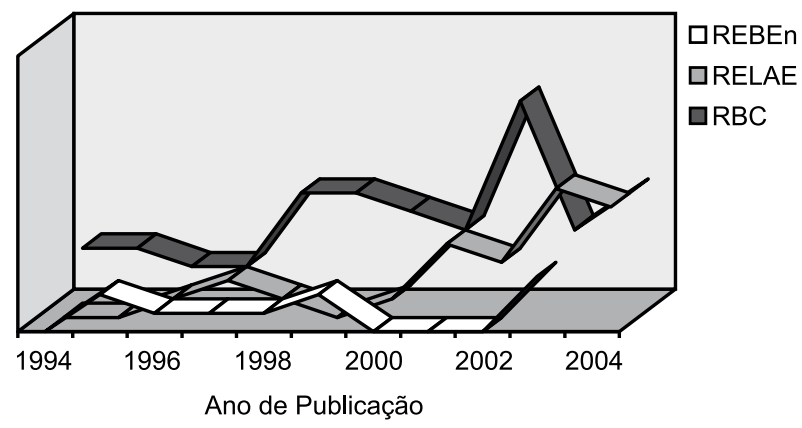

Figura 1 - Distribuição dos artigos na área da Enfermagem Oncológica publicados REBEn, RELAE e RBC (1994-2004).

Quanto a sua natureza, conforme apresentado na Figura 2, a ênfase dos artigos incide em pesquisas (64), seguindo-se artigos de revisão (11), reflexão (08), e relatos de experiência/caso (06). Cabe destacar que os estudos incluídos como pesquisas se originam da produção acadêmica (dissertações mestrado e teses de doutorado), além de projetos de pesquisa. O que sinaliza a busca pela construção de conhecimento sistematizado.

Quanto às temáticas, de acordo com a Figura 3 , os artigos focalizaram, principalmente aquelas relacionadas a bases para cuidar dos clientes e familiares (47), além de administração em enfermagem e modelos gerenciais/assistenciais (26); outros temas tratados foram ensino/formação de recursos humanos (04), pesquisa (04), políticas públicas (03) e saúde do trabalhador (03). 
Moreira MC, Camargo TC, Carvalho V, Figueirêdo CF, Rosa LD, Bolzan MF

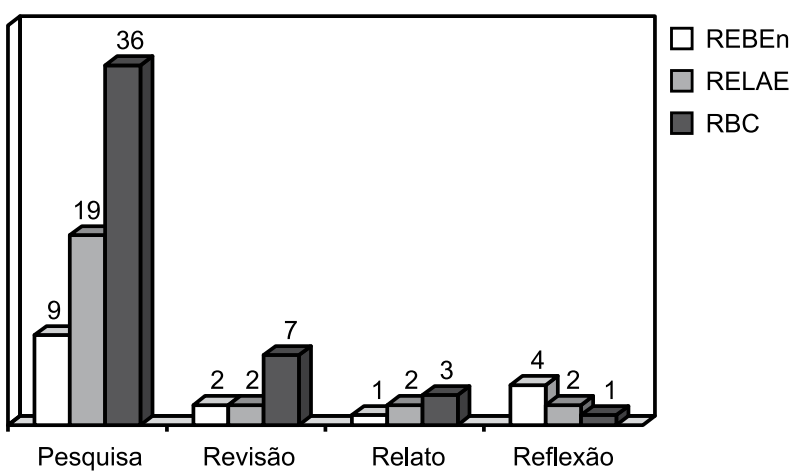

Figura 2 - Distribuição dos artigos na área de Enfermagem Oncológica de acordo com a categoria (1994-2004).

Os destaques temáticos denotam a preocupação das enfermeiras com os aspectos relacionados à assistência e à organização do processo de cuidar. $\mathrm{O}$ que sugere que estas são prioridades nas pesquisas em enfermagem oncológica no Brasil. Tendência que acompanha a produção científica da enfermagem brasileira cujo predomínio de temas estudados está relacionado às áreas assistencial e organizacional, sugerindo que a produção do conhecimento na enfermagem tem sua origem no cotidiano do trabalho, refletindo o contexto histórico social e as particularidades relativas aos avanços da profissão. ${ }^{8-9}$

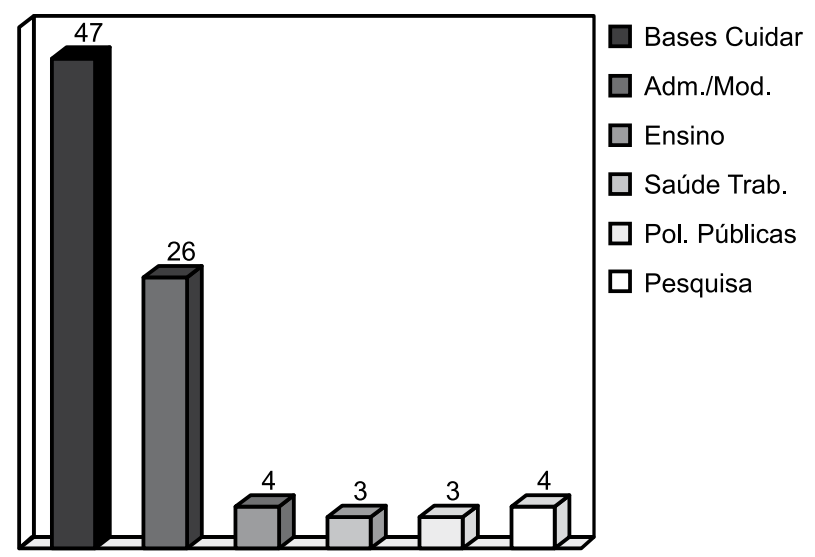

Figura 3 - Distribuição das temáticas focalizadas nos artigos publicados REBEn, RELAE e RBC na área de enfermagem oncológica (1994-2004).

Tal tendência reitera a preocupação das enfermeiras que atuam na área de oncologia em âmbito internacional. Em estudos realizados há uma década por enfermeiras americanas, holandesas e canadenses, acerca das prioridades da pesquisa em enfermagem oncológica naqueles países, já eram apontados como prioritários os estudos acerca dos cuidados de enfermagem para prevenção do câncer; manejo e controle de sintomas clínicos, além dos aspectos psicossociais e ético-legais que envolvem o cuidado. ${ }^{10-11}$

$\mathrm{Na}$ atualidade essas prioridades são ressaltadas na justificativa dos objetivos das pesquisas/publicações cadastrados na International Society of Nurses in Cancer Care (ISNCC), a qual está vinculada à Sociedade Brasileira de Enfermagem Oncológica, e cujos projetos de pesquisa em andamento estão relacionados a manejo e controle de sintomas (dor, fadiga e cuidados paliativos), além de atenção primária e educação em câncer de mama. ${ }^{12}$

Quanto a Oncology Nursing Society (ONS), um levantamento para determinar suas prioridades de pesquisa para o período de 2005 a 2008 foi realizado, considerando a enfermagem oncológica como um todo, isto é, abrangendo todas as suas áreas que incluem prevenção, detecção, tratamento, sobrevida e cuidados paliativos. A partir dos resultados, foi considerada a distribuição de fundos, da ONS e de outras organizações, destinados à pesquisa. Neste levantamento foram considerados como prioridades estudos acerca da qualidade de vida, temática já ressaltada em ranks anteriores. De outra forma vale ressaltar temas prioritários não incluídos anteriormente como; participação nas decisões no tratamento em doença avançada, educação do cliente e família, e participação nas decisões quanto ao tratamento da dor. ${ }^{13}$

Quanto às áreas que delimitam o ato de conhecer, pode-se dizer a partir dos artigos estudados, que o conhecimento produzido condiz com contribuição mais especificada, correspondendo às áreas setorizadas de enfermagem oncológica, em detrimento dos artigos relacionados a conhecimentos gerais com enfoque no ensino e pesquisa na área de oncologia. O destaque nos estudos está relacionado à área de oncologia cirúrgica (clientes laringectomizados e em outras situações de pré e pós-operatório), oncologia clínica (nas áreas de dor, quimioterapia, radioterapia e transplante de medula), além de prevenção e controle do câncer (mama e colo de útero), cuidados em pediatria e cuidados paliativos.

Pode-se dizer que há predomínio nos trabalhos pela intencionalidade objetiva pertinente a situações vivenciadas pelos clientes (67 estudos). Enquanto outros trabalhos (20) têm como foco a intenção/consciência do sujeito no conhecimento, ou seja, estudos que focalizaram como sujeitos do estudo a enfermeira/equipe de enfermagem. 
Questões epistemológicas relacionadas ao enquadramento teórico-metodológico

Neste item foram ressaltadas as questões epistemológicas relacionadas à pertinência do enquadramento teórico-metodológico adotado, considerando os referenciais utilizados no estado consagrado, sancionado ou em construção, além do tratamento dos dados. A apreensão dessas questões possibilita, a partir dos estudos analisados, identificar a autonomia e fronteiras teórico-metodológicas que configuram o campo epistêmico na enfermagem oncológica.

Conforme observa-se na Figura 4, o referencial teórico adotado nos estudos predomina como revisão de literatura com enfoque nas temáticas estudadas; bases conceituais - cultura, qualidade de vida, campo em saúde, crenças em saúde, estigma, gênero, suporte social, pedagogia problematizadora, comunicação, avaliação da qualidade da saúde, e complexidade; referenciais teórico-filosóficos a exemplo de fenomenologia, interacionismo simbólico e representação social; e teorias de enfermagem - Modelo Adaptativo de Roy, Teoria de Dorothea Orem, e a Taxonomia da NANDA.

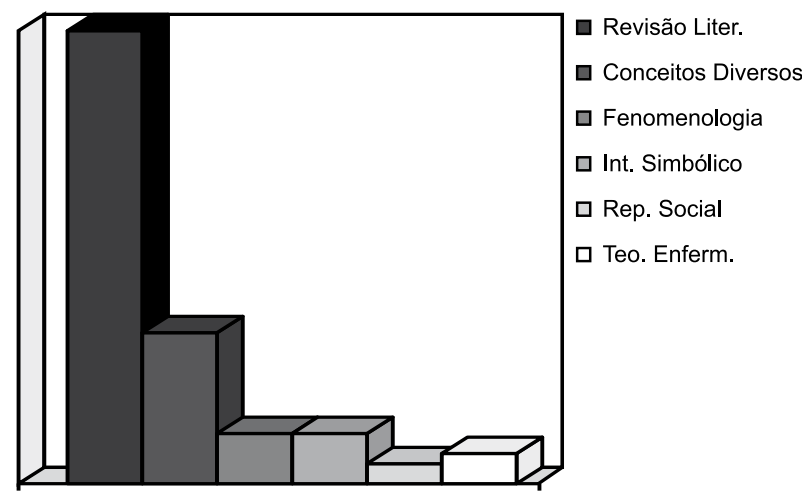

Figura 4 - Distribuição do enquadramento teórico adotado nos artigos na área de Enfermagem Oncológica REBEn, RELAE, RBC (1994-2004).

Esses referenciais indicam, por um lado, a opção das enfermeiras pela utilização de teorias consagradas e sancionadas como bases explicativas dos fenômenos estudados. De outro modo, a multiplicidade de opções teóricas sugere a complexidade dos fenômenos apreendidos na prática em enfermagem oncológica, o que requer a utilização de uma diversidade de bases explicativas dessa prática.

Em relação ao método e tratamento dos dados, verificou-se que os trabalhos, em sua maioria apresentam-se como estudos descritivos de situações- problema e estudos exploratórios; Um a freqüência menor de estudos tinha o propósito de avaliação e análise do processo assistencial.

Quanto à abordagem pode-se averiguar na figura 5 que os estudos classificam-se como: qualitativos (60), quantitativos (20) e quanti-qualitativos (08).

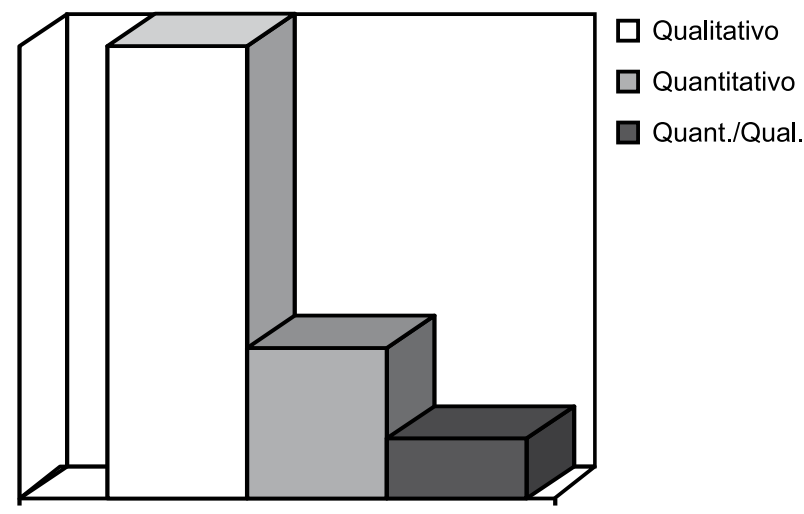

Figura 5 - Distribuição do enquadramento metodológico adotado nos artigos na Enfermagem Oncológica REBEn, RELAE, RBC (1994-2004).

\section{CONCLUSÃO}

Os objetivos deste estudo foram identificar as publicações na enfermagem em oncologia no período de 1994 a 2004 e analisar nestas publicações, os aspectos epistemológicos destacados nas temáticas focalizadas e no enquadramento teórico-metodológico adotado nos estudos, utilizando-se para tal de um referencial teórico-metodológico aliado a uma metodologia de categorização epistemológica para a pesquisa na enfermagem.

Neste sentido e à guisa de conclusão, os resultados obtidos a partir desta pesquisa possibilitaram apreender que as enfermeiras, na quase totalidade dos estudos para a construção do conhecimento, buscam respaldo em disciplinas conexas à enfermagem, principalmente na área de oncologia e das ciências sociais. No entanto, cabe ressaltar que apenas poucos estudos utilizam teorias de enfermagem como embasamento teórico.

Concluiu-se ainda, que há uma acentuada tendência para o desenvolvimento de pesquisas qualitativas, o que está de acordo com os achados de outras pesquisas nacionais que ressaltam a tendência da utilização deste tipo de pesquisa e de abordagens descritivas, ${ }^{14,15}$ que possibilitam a compreensão de vivências, emoções, sentimentos e comportamentos que permeiam o processo assistencial na enfermagem oncológica. 
Com base nesse estudo, ainda de caráter preliminar, as autoras consideram que os achados permitem anunciar a especificidade do conhecimento produzido nas dimensões e nas formalidades $\mathrm{da}$ atuação de enfermeiras(os) na área de enfermagem oncológica. O que indica que tal conhecimento é consistente com a complexidade e as tentativas de explicação sobre a arte de cuidar dos clientes com câncer e com esforços de definir/ampliar critérios e padrões assistenciais.

Apesar de não haver, nos estudos analisados, experiências sob controle ou compatíveis com regras de demonstração de evidências, a posição das autoras é definida em favor da relevância da metodologia utilizada para a elucidação da produção científica e para apontar necessidade de mudanças de indicadores de modelos gerenciais e assistenciais.

\section{REFERÊNCIAS}

1 Carvalho V. A enfermagem de saúde pública como prática social: um ponto de vista crítico sobre a formação da enfermeira em nível de graduação. Esc. Anna Nery Rev. Enferm. 1997 Jul; I (0): 25-41.

2 Japiassu H. Introdução ao pensamento epistemológico. 2a ed. Rio de Janeiro (RJ): Francisco Alves; 1977.

3 Ziman J. O Conhecimento confível: uma exploração dos fundamentos para a crença na ciência. Campinas (SP): Papirus; 1996.

4 Moreira MC, Carvalho V, Caldas TC, Gutiérrez MG. Tendências da produção de conhecimento na enfermagem oncológica no Brasil: um estudo preliminar. In: Anais do 3o Congreso de la Federación Latinoamericana de Enfermeria Oncologica; 2000 Set 21-23; Montevideo, Uruguai. Montevideo (UR): Federación Latinoamericana de Enfermeria Oncologica; 2000. p 8.

5 Carvalho V, Figueiredo NMA, Leite JL, Moreira MC. Questões epistemológicas da construção do conhecimento na enfermagem: do ensino à prática de cuidar. Esc. Anna Nery Rev. Enferm. 2003 Ago; 7 (2): 137-40.

6 Gutiérrez MGR, Vieira TT, Almeida MCP, Elsen I, Stefanelli MC. Acompanhamento e avaliação da pós-graduação no Brasil: retrospectiva histórica da representação da enfermagem. Esc. Anna Nery Rev. Enferm. 2001 Ago; 5 (2): 161-72.

7 Spagnolo F, Souza VC. O que mudar na avaliação da CAPES? Rev. Bras. Pós-Grad. 2004 Nov; 1 (2): 8-34.

8 Gutiérrez MGR, Leite JL, Pagliuca LMF, Erdman AL. Os múltiplos problemas pesquisados e a pesquisar na enfermagem. Rev. Bras. Enferm. 2002 Set-Out; 55 (5): 535-41.

9 Silveira LC, Dias MAS, Chagas IOO, Damasceno MMC, Fraga MNO. Tendências das teses de doutoramento em enfermagem produzidas na Universidade do Ceará. Texto Contexto Enferm. 2003 Jul-Set; 12 (3): 314-22.

10 Stelz KM, HabermanJH, Jones LS. Oncology Nursing Society Research Priorities Survey. Oncology Nursing Forum. 1995 Jun; 22 (5): 1-13.

11 Bakker DA, Fitch M. Oncology nursing research priorities: a Canadian perspective. Cancer Nursing. 1998 Dez; 21 (6): 394-401.

12 International Society of Nurses in Cancer Care [acesso em 2006 Abr 23]. Disponível em: http:// www.isncc.org

13 Berger AM, Berry DL, Christopher KA, Greene AL, Malisk S, Swenson KK, et al. Oncology nursing society year 2004 research priorities survey. Oncology Nursing Forum. 2005 Mar; 32 (2): 281-90.

14 Camargo TC; Souza IEO. A pesquisa em enfermagem no Instituto Nacional de Câncer: trajetória, tendências e perspectivas. Rev. Bras. Cancerologia. 2003 Jul-Set; 49 (3): 159-66.

15 Silveira CS. Pesquisa em enfermagem oncológica no Brasil: uma revisão integrativa [dissertação]. Ribeirão Preto (SP): USP/EERP; 2005. 